Philosophical Magazine A, 1984, Vol. 49, No. 1, 95-109

\title{
Elastic strains and the energy balance for multiply twinned particles
}

\author{
By A. Howie and L. D. Marks $\dagger$ \\ Cavendish Laboratory, Madingley Road, \\ - Cambridge CB3 0HE, England
}

[Received 1 June 1983 and accepted 28 June 1983]

\begin{abstract}
The energetics of multiply twinned particles (MTPB) are investigated using elasticity theory. This allows the homogeneous strain models to be critically compared with the disclination model for the strains in decahedral particles and with a new model for the strains in icosahedral particles based on inhomogeneous elasticity. The overall energy balance between MTPs and single crystals is then evaluated, including the significant cost of elastially distorting the surface and using two extreme models of the faceting. The results of this analysis indicate that icosahedral MTPs will be more stable than single crystals for small sizes only for strong faceting conditions, decahedral MTPs being true intermediaries between the two. Experimentally observed stress-relief mechanisms provide indirect evidence for the inhomogeneous strain models.
\end{abstract}

\section{§ 1. Introduction}

Multiply twinned particles (MTPs), which frequently occur in the early stages of particle growth, present an interesting problem for structural, thernodynamic and kinetic theory and may be of considerable practical significance in a variety of fields. Instead of being miniature versions of a translationally invariant solid, MTPs have instead the discrete symmetries of a non-crystallographic point group. The simplest model for MTPs is a collection of singlecrystal tetrahedra, twin related on their adjoining faces, as illustrated in fig. 1. Five such units with two twin boundaries each assemble about a common [110] axis to form a decahedral MTP, or Dh, with $\mathrm{D}_{5 \mathrm{~h}}$ symmetry ; twenty units with three twin boundaries each and a common vertex form an icosahedral MTP, or Ic, with $I_{h}$ symmetry.

Observed and analysed in detail during the early stages of epitaxial growth (Ino 1966, Ino and Ogawa 1967, Allpress and Sanders 1967), MTPs have since been found in argon smokes (see, for example, Hayashi, Ohno, Shigeki and Uyeda 1977) during electrolytic deposition (see, for example, Disgurd, Maurin and Roberts 1976) and more recently in heterogeneous silver catalysts (Marks and Howie 1979). At present it appears that MTPs can be prepared for all the f.c.c. metals (Hayashi et al. 1977) and certain related materials such as germanium or silicon (Saito, Yatsuya, Mihama and Uyeda 1978), and Dhs have been observ́ed in magnesium (Ohno and Yamauchi 1981).

$\dagger$ Present address : Department of Physics, Arizona State University, Tempe, Arizona 85287, U.S.A. 
Fig. 1

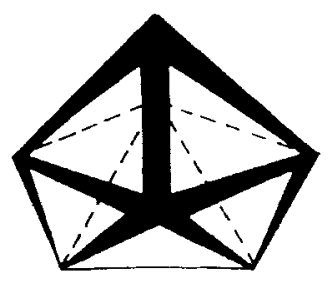

(a)

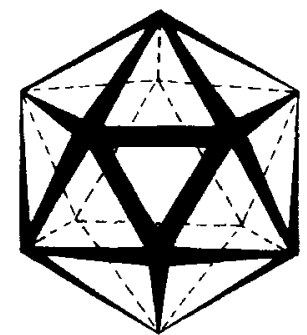

(b)

Schematic diagrams of the structure of $(a)$ decahedral and $(b)$ icosahedral MTPs.

Fig. 2

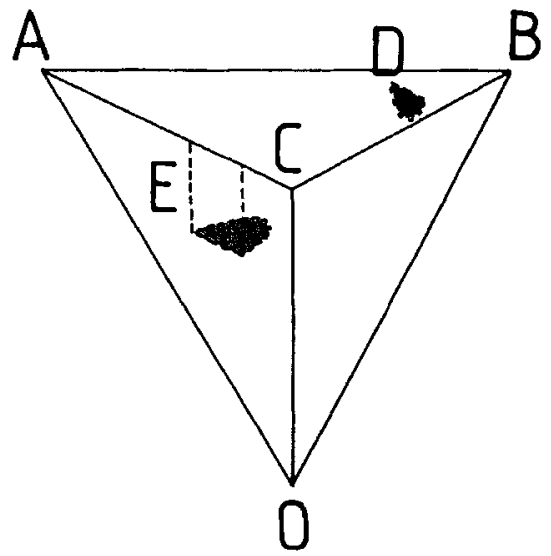

Tetrahedral segment showing favourable positions for the nucleation of a Frank loop $\mathrm{E}$ or a Shockley loop $\mathrm{D}$ as a result of tractions applied to eliminate angular gaps between segments in an MTP. For a Dh (with axis OA), normal tractions must be applied to the faces $A O B$ and $A O C$; for an Ic (with centre O), normal tractions must be applied to the faces $\mathrm{AOB}, \mathrm{BOC}$ and $\mathrm{COA}$.

When constructed from perfect single-crystal units, neither type of MTP is completely space-filling, so it is necessary to incorporate some form of internal distortion : a homogeneous elastic strain (Ino 1966), an inhomogeneous strain (de Wit 1972), dislocations (Saito et al. 1978, Marks and Howie 1979, Marks and Smith 1981, 1983) or a crystal structural modification (Bagley 1965, Heinemann, Yacaman, Yang and Poppa 1979, Yacaman, Heinemann, Yang and Poppa 1979, Yang 1979, Yang, Yacaman and Heinemann 1979). If the distortion is an internal elastic strain, it can be viewed in two different ways : the particles can be either dealt with as complete entities or decomposed into their single-crystal units. Treating the single crystals separately for both MTPs, the strains are equivalent to pulling on the twin boundaries, as illustrated in fig. 2. The sense of this distortion is to an orthorhombic crystal structure for Dhs and a rhombic structure for Ics. Alternatively, a complete Dh contains a screw disclination, as pointed out by de Wit (1972), whilst an Ic contains six disclinations. 
The surface energy contribution, which favours small MTPs relative to single-crystal Wulff polyhedra, has been discussed in a companion paper by Marks (1984, hereafter referred to as Paper I). In the Ics the favourable factor is primarily the predominance of low-energy (111) facets which define an approximately spherical particle. The situation is more complicated in Dhs where, in addition to the (111) and (100) facets considered previously by other authors, there are large, slightly re-entrant (111) facets at the twin boundaries. These play a crucial role both in leading to an approximately spherical particle and in lowering the total surface energy below that of a single crystal.

In this paper we investigate further the energetics of MTPs, working mainly within the framework of elasticity theory. This approach may be regarded as complementary to the potentially more rigorous methods based on direct quantum-mechanical calculation of cluster energies, or on models using some assumed interatomic potential--elasticity theory contains rather fewer unknown parameters and is expected to be reasonably reliable in large particles where other methods become excessively laborious. In $\S 2$ we give a critical comparison of the homogeneous strain models and of the disclination solution (for a $\mathrm{Dh}$ ) given by de Wit (1972). The analysis is then extended to Ics, using a new model for the strains based on inhomogeneous elasticity. An important energy term neglected in all previous analyses, namely the cost of elastically distorting the surface, is then treated in $\$ 3$. The overall energy balance between MTPs and single crystals is evaluated in $\S 4$ for two extreme models of faceting (analysed previously in Paper I). In $\$ 5$ stress-relief mechanisms are briefly examined in the light of recent experimental results, and a general discussion is presented in $\S 6$.

\section{§ 2. Internal ELASTIC STRAIN Energies}

\subsection{Homogeneous elastic strains}

The simplest model of the internal distortions is a homogeneous elastic strain with linear displacements within each single-crystal unit of the MTP (Ino I969). The solution can be found here by minimizing the strain energy in the particles, subject to the constraint of filling up the angular gaps. For the Dh, the displacements parallel to the symmetry axis vanish, but there is a tensile strain $\frac{1}{2} \epsilon_{D}^{\prime}$ in the [110] direction parallel to the outer perimeter, and an equal compressive strain in the axial direction at right angles to this where $\epsilon_{D}^{\prime}=0.027485$. For the Ic we find compressions normal to the outer face of each unit of $\frac{2}{3} \epsilon^{\prime}{ }_{I}$, and tensile strains of $\frac{1}{3} \epsilon^{\prime}{ }_{I}$ in each of the two perpendicular directions, where $\epsilon_{I}^{\prime}=0.080362$. (The cubic dilatation vanishes in both cases.) Values for the strain energy densities for isotropic elastic constants are given in table 1 .

Homogeneous solutions satisfying the constraints can also be created with uniform angular and radial strains using cylindrical or spherical geometries approximating a complete $\mathrm{Dh}$ or Ic, respectively. For a $\mathrm{Dh}$ we find that $2 e_{\theta \theta}=-2 e_{\rho \rho}=\epsilon_{\mathrm{D}}=0.0205$, and for an Ic that $3 e_{\theta \theta}=3 e_{\phi \phi}=-3 e_{r r} / 2=\epsilon_{\mathrm{I}}=0.0615$. Compared with the linear displacements discussed above, these strains and the strain energies are appreciably smaller, as can be seen from table 1 . 
Table 1. Isotropic elastic strain energy densities for the different models for MTPs. Here $\mu$ is the shear modulus and $\nu$ Poisson's ratio, $\epsilon_{D}^{\prime}=0.0275, \epsilon_{D}=0.0205$, $\epsilon_{\mathrm{I}}^{\prime}=0.0804$ and $\epsilon_{\mathrm{I}}=0.0615$.

\begin{tabular}{lc}
\hline \multicolumn{1}{c}{ Model } & Strain energy density \\
\hline Dh & \\
Linear homogeneous & $\frac{1}{2} \mu \epsilon_{\mathrm{D}}^{\prime}{ }^{2}$ \\
Angular and radial homogeneous & $\frac{1}{2} \mu \epsilon_{\mathrm{D}}^{2}$ \\
Disclination in plane strain & $\frac{1}{4} \mu \epsilon_{\mathrm{D}}^{2} /(1-\nu)$ \\
Ic & $\frac{2}{3} \mu \epsilon_{\mathrm{I}}^{\prime}{ }^{2}$ \\
Linear homogeneous & $\frac{2}{3} \mu \epsilon_{\mathrm{I}}^{2}$ \\
Angular and radial homogeneous & $\frac{2 \mu \epsilon_{\mathrm{I}}^{2}}{9}\left(\frac{1+\nu}{1-\nu}\right)$ \\
Angular and radial inhomogeneous & \\
\hline
\end{tabular}

Both these models leave unbalanced tractions at the external surface and across the twin interfaces between the units for the linear models. Relief of the imbalance leads to inhomogeneous strain solutions, as described below, and a further significant reduction in the elastic strain energy.

\subsection{Inhomogeneous strains in Dhs}

A solution for the inhomogeneous strains in a Dh has been given by de Wit (1972), based on the original Volterra (1907) solution for a disclination. De Wit approximated the $\mathrm{Dh}$ by a section of a cylinder in plane strain, for which (with cylindrical polar coordinates and the curved surface free from external loads) the displacements and strains are given by

$$
\begin{aligned}
u_{\rho} & =\left[\frac{1-2 \nu}{2(1-\nu)}\right] \epsilon_{\mathrm{D}} \rho \ln (\rho / R)-\epsilon_{\mathrm{D}} \rho / 2, \\
u_{\phi} & =\rho \epsilon_{\mathrm{D}} \\
e_{\rho \rho} & =\left[\frac{1-2 \nu}{2(1-\nu)}\right] \epsilon_{\mathrm{D}}[\ln (\rho / R)+1]-\epsilon_{\mathrm{D}} / 2, \\
e_{\phi \phi} & =\left[\frac{1-2 \nu}{2(1-\nu)}\right] \epsilon_{\mathrm{D}} \ln (\rho / R)+\epsilon_{\mathrm{D}} / 2, \\
e_{z z} & =e_{\rho z}=e_{z \phi}=e_{\phi \rho}=0,
\end{aligned}
$$

and the stress components by

$$
\begin{aligned}
\sigma_{\rho \rho} & =2 \mu \epsilon_{\mathrm{D}} \ln (\rho / R), \\
\sigma_{\phi \phi} & =\frac{\mu \epsilon_{\mathrm{D}}}{1-\nu}[\ln (\rho / R)+1], \\
\sigma_{z z} & =\frac{\mu \nu \epsilon_{\mathrm{D}}}{1-\nu}[2 \ln (\rho / R)+1], \\
\sigma_{\rho z} & =\sigma_{z \phi}=\sigma_{\phi \rho}=0,
\end{aligned}
$$


where $R$ is the radius of the cylinder, $\nu$ Poisson's ratio and $\mu$ the shear modulus. These strains correspond to a high compression near the axis and a dilatation near the outer perimeter of the particle. There is, therefore, a strain energy associated with the distortion, given by

$$
W=\frac{\mu \epsilon_{\mathrm{D}}{ }^{2}}{4(1-\nu)} V
$$

where $V$ is the volume of the particle (it should be noted that $W$ is proportional to the volume of the particle). Assuming a Poisson's ratio of $\frac{1}{3}$, this is only $75 \%$ of the lowest homogeneous strain energy density (table 1).

These strains become infinite along the core of the disclination ( $z$ axis), to avoid which de Wit (1972) introduced an axial cut-off. Unlike what happens with a dislocation core, however, such a cut-off is unnecessary to secure the convergence of the strain energy integral. Furthermore, the strains in the core are unreasonable only for rather large particles, where extensive strain relief through dislocations is likely. For small particles the strains are not unreasonable at an atomic spacing from the axis. For example, $0.1 \mathrm{~nm}$ from the centre of a $\mathrm{Dh}$ of $10 \mathrm{~nm}$ radius, the cubic dilatation is only $3.5 \%$. We therefore avoid the use of a cut-off here.

The inhomogeneous strains given here are only approximations to the true solutions. They apply strictly to an infinitely long cylinder, and not for the rather spherical shape of the particles. In principle, further radial strains could be superimposed and the strain energy integral minimized over, for example, a sphere. However, such additional complications seem unwarranted unless displacements in the $z$ direction are also included, and an evaluation of these is intractable without introducing displacements that are singular along the $z$ axis. Consequently, we use the simple strain-energy relationship given above, which should represent a better estimate than values derived with a purely homogeneous strain.

\subsection{Inhomogeneous strains in Ics}

The strains in an Ic can be approximated if we replace the Ic by an elastic sphere having the same missing volume (about $12 \%$ ), but spread uniformly throughout the sphere. This is equivalent to an angular average of the strains, and may be visualized as a multitude of thin radial cones, each subtending a very small solid angle $d \omega$ at the centre, with small angular gaps between them in the unstrained state. The cones are now constrained by external forces to remain of the same length $R$ while they are distorted sideways until they touch, and are then ' glued ' together. In the limit $d \omega \rightarrow 0$, the strain is uniform throughout the sphere and is described by the relationships

$$
\begin{aligned}
& e_{\theta \theta}=e_{\phi \phi}=\epsilon_{1}, \\
& e_{\theta \phi}=e_{\phi r}=e_{r \theta}=e_{r r}=0,
\end{aligned}
$$

(It can be shown that these strains represent mean values averaged over the solid angle of the particle.) On removing the restraints the continuous sphere 
now distorts radially to an equilibrium state. The full strain components are thus of the form (E. H. Yoffe 1980, private communication)

$$
\begin{gathered}
e_{r r}=\frac{\partial u r}{\partial r} \\
e_{\theta \theta}=e_{\phi \phi}=\frac{u_{r}}{r}+\epsilon_{\mathrm{I}} .
\end{gathered}
$$

The stress components derived from these must satisfy the equilibrium equation

$$
\frac{\partial \sigma_{r r}}{\partial r}+\frac{2 \sigma_{r r}-\sigma_{\theta \theta}-\sigma_{\phi \phi}}{r}=0
$$

On substitution, this gives for $u_{r}$ the differential equation

$$
\frac{\partial}{\partial r}\left[\frac{1}{r^{2}} \frac{\partial}{\partial_{r}}\left(r u_{r}\right)\right]=\frac{2 \epsilon_{\mathrm{I}}}{r}\left(\frac{1-2 \nu}{1-\nu}\right),
$$

which has the general solution

$$
u_{r}=\frac{2 \epsilon_{1}}{3}\left(\frac{1-2 \nu}{1-\nu}\right) r \ln r+B r+C / r^{2}
$$

As the radial displacements must vanish at the centre of the sphere, and the external surface must be free of external forces, then

$$
\left.\begin{array}{l}
C=0, \\
B=\frac{2}{3} \epsilon_{\mathrm{I}}\left(\frac{1-2 \nu}{1-\nu}\right) \ln R-\frac{2}{3} \epsilon_{1} \cdot
\end{array}\right\}
$$

The strains and stresses are therefore given by

$$
\begin{aligned}
& e_{r \tau}=\frac{2}{3} \epsilon_{\mathrm{I}}\left(\frac{1-2 \nu}{1-v}\right)\left[\ln \left(\frac{r}{R}\right)+1\right]-\frac{2 \epsilon_{\mathrm{I}}}{3}, \\
& e_{\theta \theta}=e_{\phi \phi}=\frac{2 \epsilon_{\mathrm{I}}}{3}\left(\frac{1-2 v}{1-\nu}\right) \ln \left(\frac{r}{R}\right)+\frac{\epsilon_{\mathrm{I}}}{3} \\
& \sigma_{r \tau}=\frac{4 \mu \epsilon_{\mathrm{I}}}{3}\left(\frac{1+\nu}{1-\nu}\right) \ln \left(\frac{r}{R}\right) \\
& \sigma_{\theta \theta}=\sigma_{\phi \phi}=\sigma_{r r}+\frac{2 \mu \epsilon_{\mathrm{I}}}{3}\left(\frac{1+\nu}{1-\nu}\right)
\end{aligned}
$$

whilst the strain energy of the distortion is

$$
W=\frac{2 \mu \epsilon_{\mathrm{D}}^{2}}{9}\left(\frac{1+\nu}{1-\nu}\right) V,
$$

$V$ again being the volume of the particle. Assuming again a Poisson's ratio of $\frac{1}{3}$, this is $67 \%$ of the homogeneous strain energy.

As with the Dhs, the stresses are singular in the compressive region at the origin, but a central cut-off is a superfluous complication. The strain and total strain energy values derived are only approximate. The use of a spherical 
shape for the Ics should be advantageous, but our use of an angular average for $e_{\theta \theta}$ and $e_{\phi \phi}$ will introduce errors. However, the incorporation of an inhomogeneous radial displacement will almost certainly ensure that a better value of the strain energy is produced than with a simple homogeneous strain.

\section{§ 3. Surface Elastic STrain ENERGY}

To obtain a realistic estimate of the energy balance between single crystals and MTPs, a further energy term must be considered--the energy required to strain the surface. This may be regarded as a correction to the total surface energies given in Paper I which were based on surface free energies of unstrained surfaces. Although this term is far smaller than the total surface energies of the particles, it is comparable to the differences between them, and larger than the total twin energies. The correction term can be expressed formally by expanding the surface free energy per unit area (of the unstrained state) in the form

$$
\gamma=\gamma_{\mathrm{s}}+\sum_{i j} e_{i j} \frac{\partial \gamma}{\partial e_{i j}}+\ldots
$$

where the strain variation is described (to first order) by the 'surface stress tensor' $g_{i j}(\theta, \phi)$ of the material, given by

$$
g_{i j}(\theta, \phi)=\frac{\partial \gamma(\theta, \phi)}{\partial e_{i j}}
$$

where $i$ and $j$ are taken over the directions in the surface plane. We have deliberately adopted here the older definition of $g_{i j}(\theta, \phi)$ in terms of the number of atoms involved rather than the final surface area (Royal Society 1969). Our definition is preferable for small metal particles as it is consistent with standard elasticity and avoids any complicated renormalizations to ensure that the particle volumes used refer to the number of atoms involved. The energy term to be added is

$$
\Delta W=\sum_{i j} \int e_{i j} g_{i j} d S
$$

We make the simplifying approximation

$$
g_{i j}(\theta, \phi)=g \delta_{i j} \gamma_{\mathrm{B}}(\theta, \phi),
$$

where $g$ is a constant whose value will be discussed later. It is now possible to use the modified Wulff theory for MTPs, described in Paper I, to reduce the integrals, i.e.

$$
\begin{aligned}
\Delta W & =\sum_{i j} \int e_{i j} g_{i j}(\theta, \phi) d S=g \sum_{i} \int e_{i i} \gamma_{s}(\theta, \phi) d S \\
& =g \bar{e}_{\mathrm{s}} \int \gamma_{\mathrm{s}}(\theta, \phi) d S \\
& =g \bar{e}_{\mathrm{s}} \gamma_{111} \epsilon_{\omega} V^{2 / 3}
\end{aligned}
$$


where $\epsilon_{\omega}$ is the dimensionless parameter described in eqn. (36), $\bar{e}_{\mathrm{s}}$ the sum of the total strains in the surface and $\gamma_{111}$ the surface free energy per unit area of an unstrained (111) face. (Throughout this paper we will use a definition of the volume $V$ of the particles in terms of the unstrained volume, i.e. the number of atoms involved.) From the strain solutions given in $\S 2$,

$$
\begin{aligned}
& e_{8}=\frac{2}{3} \epsilon_{\mathrm{I}} \text { for an Ic, } \\
& e_{\mathrm{s}}=\frac{1}{2} \epsilon_{\mathrm{D}} \text { for a } \mathrm{Dh} .
\end{aligned}
$$

So far, we have evaluated only the surface cost of the distortion, and the surface stress will in general also exert an effective compressive or expansive load on the surface under which a small particle will relax still further. In linear elastic theory (Eshelby 1952, 1956) these changes are independent of any equilibriated stresses already existing in the particle, but do depend on the shape of the external surface, and can therefore be different in single crystals, Dhs or Ics. Assuming a homogeneous strain and using the approximations for the surface integrals given above, we find an extra homogeneous strain

$$
\Delta e_{r r}=\Delta e_{\phi \phi}=\Delta e_{\theta \theta}=-\frac{\epsilon_{\omega} g \gamma_{111}}{3 V^{1 / 3} \mu}\left(\frac{1-2 \nu}{2}\right)
$$

and a free energy change of

$$
\Delta G=-\frac{\epsilon_{\omega}{ }^{2} g^{2} \gamma_{111} V^{1 / 3}}{3}\left(\frac{1-2 \nu}{2}\right)
$$

The effect of the term $\Delta G$ on the energy balance between MTPs and single crystals is very small, and we include it only for completeness.

The values of $g_{i j}(\theta, \phi)$ for metals are not known at present. In principle they might be obtained from the lattice contractions of small particles (see, for example, Vermaak, Mays and Kuhlmann-Wilsdorf 1968). However, quantitative interpretation of these results is problematic as lattice changes due to surface relaxations, internal defects such as dislocations or, indeed, MTPs could also be present. For example, surface relaxations can be modelled by a thin surface shell of abuormal lattice parameter. This yields an average lattice parameter change inversely proportional to the particle radius, identical in form to the effect attributed to the surface stresses. In principle it should be possible to calculate $g_{i j}(\theta, \phi)$ using, for instance, pseudopotential theory, and it appears likely that both positive and negative values can occur. For rare gases with simple Lennard-Jones potentials, for instance, the effect of the more distant neighbours in the bulk is to compress the nearest-neighbour separation below that of a diatomic molecule. The absence of many of these long-range interactions near the surface will therefore favour a net expansion corresponding to negative $g$. For metals, it is unclear what form of surface potential should be used, or even how valid the model is. However, it is suggestive that the surfaces of many clean metals reconstruct, a process which can be interpreted as elastic buckling to relieve a negative $g$. Further work, and experimental data under controlled conditions of surface cleanness (to eliminate any suspicions of impurity anomalies), would be of interest. 


\section{§. Energy balance}

We are now in a position to analyse the overall energy balance between the different particles. The total free energy can be expressed in the form

$$
G=-\frac{\epsilon_{\omega}^{2} g^{2} \gamma_{111}{ }^{2} V^{1 / 3}}{6 \mu}\left(\frac{1-2 \nu}{1+\nu}\right)+\epsilon_{\omega} \gamma_{111} V^{2 / 3}\left(1+g \bar{e}_{s}\right)+W V+H(V),
$$

where $\epsilon_{\omega}$ is the dimensionless parameter analysed in Paper I defined by

$$
\epsilon_{\omega}=\frac{1}{\gamma_{111} V^{2 / 3}} \int \gamma_{s}(\theta, \phi) d S,
$$

and $H(V)$ includes the residual terms which are independent of shape or strain (e.g. the cohesive energy). The difference between the free energies of two different particles can be written as

$$
\Delta G=-\frac{\gamma_{111}^{2} V^{1 / 3} g^{2}}{6 \mu}\left(\frac{1-2 \nu}{1+\nu}\right) \Delta\left(\epsilon_{\omega}^{2}\right)+\gamma_{111} V^{2 / 3} \Delta\left[\epsilon_{\omega}\left(1+g \bar{e}_{\mathrm{s}}\right)\right]+V \Delta W,
$$

and will be negative so that type $a$ is more stable than type $b$ if

$$
V^{1 / 3}<R_{a, b}\left(\frac{4 \pi}{3}\right)^{1 / 3}
$$

where

$$
R_{a, b}=\frac{\gamma_{111}}{2}\left(\frac{3}{4 \pi}\right)^{1 / 3} \frac{\Delta\left[\epsilon_{\omega}\left(1+g \bar{e}_{\mathrm{s}}\right)\right]}{\Delta W}\left\{1 \pm\left[1-\frac{4 g^{2} \Delta\left(\epsilon_{\omega}{ }^{2}\right) \Delta W}{\Delta\left[\epsilon_{\omega}\left(1+g \bar{e}_{\mathrm{s}}\right)\right]^{2} 6 \mu}\left(\frac{1-2 \nu}{1+\nu}\right)\right]^{1 / 2}\right\} \text {. }
$$

Numerical values for these 'cross-over radii' $R_{a, b}$ are given in table 2 , evaluated with $g=1$ for the five f.c.c. metals for which data are available. We have considered here only the larger values of $R_{a, b}$, corresponding to the

\begin{tabular}{|c|c|c|c|c|c|}
\hline & \multicolumn{5}{|c|}{ Cross-over radius } \\
\hline & $\mathrm{Ag}$ & $\mathrm{Al}$ & $\mathrm{Au}$ & $\mathrm{Cu}$ & $\mathrm{Ni}$ \\
\hline \multicolumn{6}{|c|}{ Strong faceting model } \\
\hline$R_{\mathrm{Sc}, \mathrm{Dh}}$ & $4 \cdot 7$ & $-\mathrm{ve}$ & $5 \cdot 6$ & $2 \cdot 0$ & - ve \\
\hline$R_{\mathrm{Sc}, \text { Ic }}$ & $8 \cdot 8$ & $3 \cdot 68$ & $9 \cdot 9$ & $7 \cdot 3$ & $5 \cdot 6$ \\
\hline$R_{\mathrm{Dh}, \mathrm{Ic}}$ & $9 \cdot 2$ & $6 \cdot 39$ & $10 \cdot 3$ & $7 \cdot 8$ & $6 \cdot 5$ \\
\hline \multicolumn{6}{|c|}{ Isotropic faceting model } \\
\hline$R_{\mathrm{Sc}, \mathrm{Dh}}$ & $-\mathrm{ve}$ & -ve & -ve & $-\mathrm{ve}$ & $-v e$ \\
\hline$R_{\mathrm{Sc}, \mathrm{Ic}}$ & $-\mathrm{ve}$ & - ve & $-v e$ & - ve & $-v e$ \\
\hline$R_{\mathrm{Dh}, \mathrm{Ic}}$ & $0 \cdot 5$ & $-\mathrm{ve}$ & $0 \cdot 3$ & 0.0 & - ve \\
\hline
\end{tabular}
positive square root and to particle radii where the elasticity may be regarded as reasonably valid. Two models for the faceting of the particles have been used : a strong faceting model where only (111) and (100) surfaces are present,

Table 2. Cross-over radii for five different f.c.c. elements, expressed in nanometres. 
Table 3. The different energy terms involved in the energy balance. The values of $\gamma_{111}$ were extrapolated to $300^{\circ} \mathrm{C}$ from those given by Linford (1973), using a surface entropy of $1.2 \mathrm{~mJ} \mathrm{~m}^{-2} \mathrm{~K}^{-1}$ (Chadwick and Kirchner 1970). Twin boundary energies $\left(\gamma_{t}\right)$ which were required to caleulate the $\epsilon_{\omega}$ parameters were taken as half the stacking fault energies given by Coulomb (1978).

\begin{tabular}{crcccc}
\hline & $\mathrm{Ag}$ & $\mathrm{Al}$ & $\mathrm{Au}$ & $\mathrm{Cu}$ & $\mathrm{Ni}$ \\
\hline$\gamma_{111}\left(\mathrm{~J} \mathrm{~m}^{-2}\right)$ & $1 \cdot 9$ & $0 \cdot 996$ & $2 \cdot 26$ & $2 \cdot 51$ & $2 \cdot 96$ \\
$\gamma\left(\mathrm{mJ} \mathrm{m}^{-2}\right)$ & $10 \cdot 0$ & $62 \cdot 5$ & $17 \cdot 5$ & $22 \cdot 5$ & $62 \cdot 5$ \\
\hline$\epsilon_{\omega}\left(1+g \bar{e}_{\mathrm{s}}\right)$, strong faceting model & & & & \\
Single crystal & $5 \cdot 50$ & $5 \cdot 50$ & $5 \cdot 50$ & $5 \cdot 50$ & $5 \cdot 50$ \\
Dh & $5 \cdot 48$ & $5 \cdot 65$ & $5 \cdot 48$ & $5 \cdot 49$ & $5 \cdot 52$ \\
Ic & $5 \cdot 09$ & $5 \cdot 23$ & $5 \cdot 09$ & $5 \cdot 10$ & $5 \cdot 13$ \\
\hline$\epsilon_{\omega}\left(1+g \bar{e}_{s}\right)$, isotropic faceting model & & & & \\
Single crystal & $4 \cdot 84$ & $4 \cdot 84$ & $4 \cdot 84$ & $4 \cdot 84$ & $4 \cdot 84$ \\
Dh & $4 \cdot 87$ & $5 \cdot 05$ & $4 \cdot 88$ & $4 \cdot 88$ & $4 \cdot 92$ \\
Ie & $4 \cdot 85$ & $5 \cdot 25$ & $4 \cdot 87$ & $4 \cdot 88$ & $4 \cdot 96$ \\
$\omega_{\mathrm{Dh}}\left(10^{6} \mathrm{~Pa}\right)$ & $5 \cdot 0$ & $4 \cdot 2$ & $5 \cdot 0$ & $7 \cdot 9$ & $11 \cdot 5$ \\
$\omega_{\mathrm{Ic}}\left(10^{6} \mathrm{~Pa}\right)$ & $54 \cdot 6$ & $44 \cdot 8$ & $58 \cdot 0$ & $85 \cdot 5$ & $121 \cdot 0$ \\
\hline
\end{tabular}

and an isotropic model where the surface energy per unit area is the same for all facets. (Details of the shapes to which these two models correspond are given in Paper I.) Numerical values for $\epsilon_{\omega}\left(1+g \bar{e}_{\mathrm{s}}\right)$ for $g=1$, obtained as described in Paper $\mathrm{I}$, are given in table 3, together with values for $\gamma_{111}, W_{\mathrm{Dh}}$ and $W_{\text {Ic }}$.

The expression for the cross-over radii is dominated by the term $\Delta\left[\epsilon_{\omega}(I+\right.$ $\left.\left.g \bar{e}_{\mathrm{s}}\right)\right]$. This term, which includes the effects of faceting, the twin boundaries and the surface stresses, is highly variable, and determines the sign of the crossover radii ; if it is negative, MTPs are always unstable. Large variations occur between different metals, different models for the faceting of the particles and different values of $g$. One new feature is the position of the Dhs, particularly in the strong faceting model. As a consequence of the improved treatment for the surfaces of these particles presented in Paper $I$, they are now intermediate between single crystals and Ics. Our numerical results do not predict any true stability for Dhs, but this result should not be interpreted too strictly; minor adjustments arising from ambiguities in the model, for instance the value of $g$, will very easily lead to a stability range for these particles. Another important and logical feature is that MTPs are unstable within the isotropic faceting model. Generally, previous models have led to the conclusion that small Ics at least will always be more stable than single crystals. The result with the isotropic model explains the experimental finding that they are not always present--experimental conditions affect MTPs.

Since there is such a large difference between the prediction of the two faceting models and with varying $g$, the results should be interpreted only as 
indicating the conditions favourable for MTP formation-extensive faceting, small values of the twin boundary energy and small-to-negative surface stresses.

\section{§ 5. STRESS-RELIEF MECHANISMS}

In the preceding sections we have dealt with MTPs by assuming them to be free of any internal defects such as dislocations, with the angular gaps accommodated by inhomogeneous elastic strains. The calculated strains are large, however, so the possibility arises that stress-relief processes may occur that involve the generation of point defects or the formation of dislocation loops.

The simplest defects that can arise are point defects. For both the MTPs, the cubic dilatation is tensile at the surface, decreasing logarithmically and changing sign towards the centres. Hence a driving force exists for vacancies to migrate into the particles, and interstitials to migrate away from the centre. However, isolated point defects can be generated only at the particle surface where, at least for the Ic, the stresses inhibit vacancy formation. Vacancyinterstitial pairs could, of course, be generated in the interior and separated by migration or by the punching of prismatic dislocation loops. So far, however, no obvious direct evidence of such processes has been observed.

Recently, direct experimental evidence has been obtained (Marks and Smith 1983) for stress mechanisms involving dislocation climb or glide. By analogy with the dislocation mechanisms of stress relief in pseudomorphic epitaxial overlayers (see, for example, Cherns and Stowell 1975), the most likely processes would appear to be nucleation at the surface of either Frank or Shockley dislocation half-loops, which then respectively climb or glide on appropriate (111) planes into the interior of the particle. From the stressfield solutions obtained above (eqns. (6)-(9), (21) and (22)), conditions for the Shockley process would be most favourable on slip planes inclined at $\sim 45^{\circ}$ to the radial direction in the particle, i.e. at $\mathrm{D}$ in fig. 2. Nucleation of the Frank half-loop would be most likely on slip planes close to the radial direction, i.e. at $\mathrm{E}$ in fig. 2. Indeed, nucleation on the plane adjacent to the twin boundary would remove the cost of generating a stacking fault within the half-loop. More detailed numerical modelling is unfortunately difficult for such processes. Except for larger particles, the strain relief introduced by a dislocation is not small relative to the total angular defect in the particle, so any complete evaluation of the energetics requires a comprehensive analysis of the interaction between the particle strain field and that of the dislocation.

Recently, direct experimental evidence has been obtained in the Ic (Marks and Smith 1983) for the operation of the Frank climb mechanism described above followed by a Shockley glide process on a slip plane parallel to the surface (see fig. 3). This is at least consistent with the inhomogeneous strain solution given in $\$ 2.3$, since eqn. (22) shows that climb will occur down to a radius $r \simeq 0.7 R$, where $\sigma_{\theta \theta}$ changes sign. The shear stresses now favour conversion of the Frank partial into a stair-rod and a Shockley partial which glides across a plane at this radius and is parallel to the external surface, in agreement with the observations (see figs. 3 and 4 ). In principle the reaction could proceed across the whole of this plane, and also on all the surrounding twin boundaries, but there is no evidence of this so far available. For completeness we also show, in fig. 5, a very small defect observed near the surface 
of an Ic where distortions are evident both inside the particle and at the twin boundary. This electron micrograph suggests that the detailed core structure of the defect in Ics is complicated, the effective Frank partial dislocation perhaps being dissociated to some extent into stair-rod and Shockley pairs even at the earliest stages of nucleation. (As such, the defect structure would be related to the dislocation trigons observed by Cherns and Stowell (1975).)

For the Dhs the considerably smaller stress levels will make dislocation loop formation much harder than in Ics. It is significant that most experimental observations of Dhs, particularly relatively small ones, have produced

Fig. 3

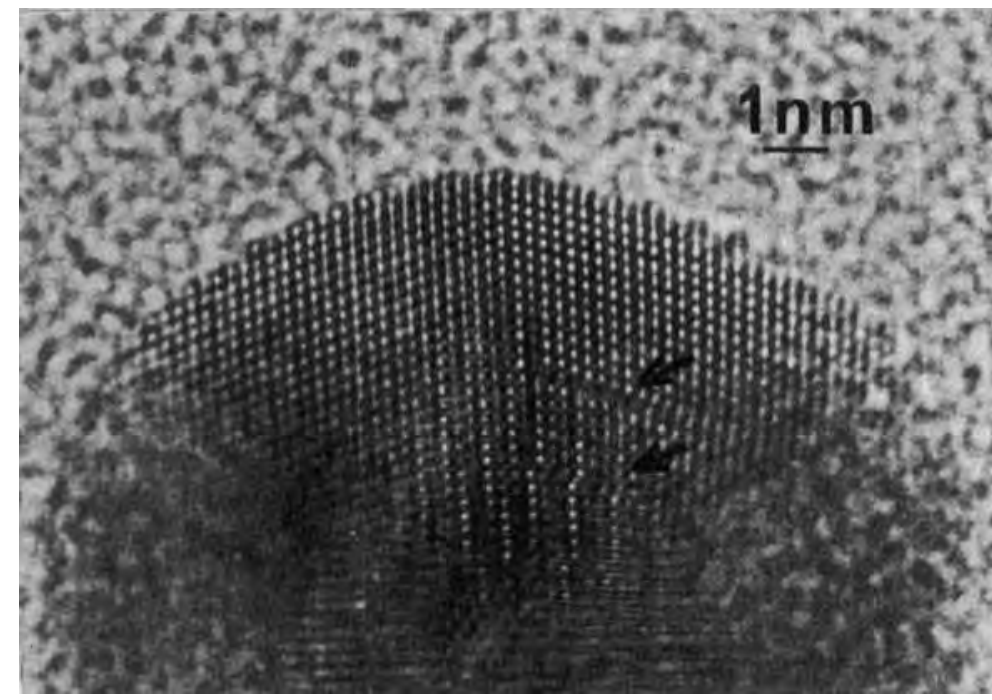

Electron micrograph showing defects in a small gold Ic. In this image the columns of atoms show black-white contrast. Two dissociated Frank partials are arrowed.

Fig. 4

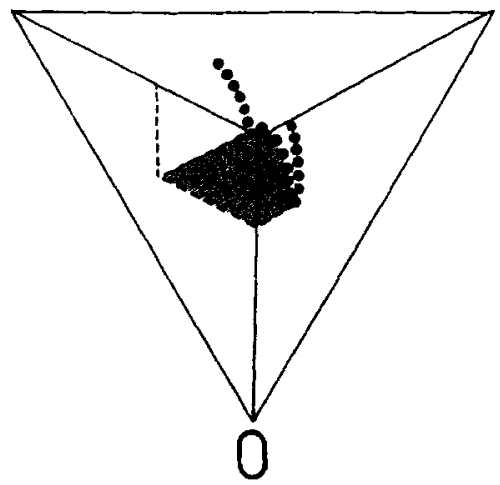

Diagram illustrating the decomposition of a Frank dislocation in an Ic into Shockley and stair-rod partial dislocation pairs. Shockley partials are indicated by the dotted lines, and stair-rods by dashed lines, and the experimentally observed stacking fault is shaded. 
Fig. 5

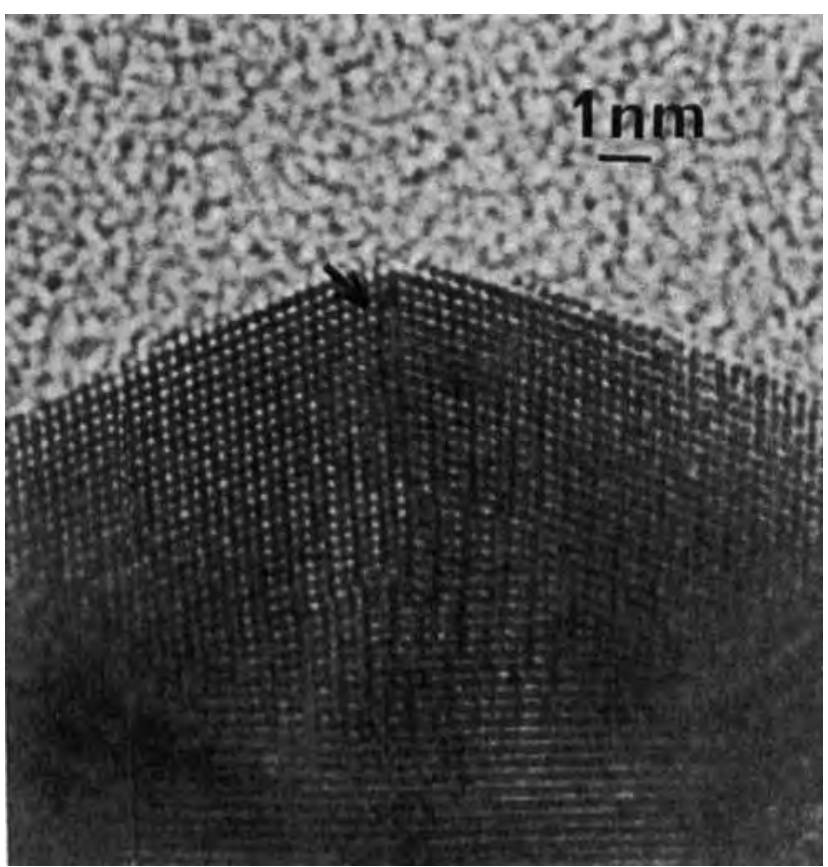

Electron micrograph showing black-white contrast for columns of atoms and a defect (arrowed) near the surface of a small gold Ic. Distortions in the lattice are evident both at the twin boundary and slightly further into the segment; surface steps are also visible.

no evidence for dislocation loops comparable to those in Ics. The consistent defect structure observed in relatively large particles (Fukano and Wayman 1969) and more recently in small particles (Marks and Smith 1983) is stacking faults parallel and near (but not necessarily atomically adjacent to) the twin boundaries. These correlate with a process first described by de Wit (1969). In a continuum model, the climb of an edge dislocation into a $\mathrm{Dh}$ to the central axis corresponds to an effective migration of the disclination towards the particle surface. The experimental observation of this defect interaction mechanism between a dislocation and the disclination does provide indirect evidence for the inhomogeneous strain model.

\section{§. Discussion}

The main intention in this paper has been to investigate the implications of inhomogeneous elastic strains in MTPs. Our results indicate that they lead to rather large reductions in the stored elastic strain energy, and are consistent with the stress-relief mechanisms that have been observed experimentally. Some details of the thermodynamics of small particles are still unclear (particularly the surface stress contribution), but a number of general trends are now apparent. The formation of MTPs is favoured by extensive faceting, small twin boundary energies, and small or negative surface stresses. If 
sufficient of these conditions are met, Ics will be the most stable form for very small particles, with Dhs either metastable intermediaries or stable in their own right over a narrow size range. However, in many cases MTPs will be thermodynamically unstable. In agreement with the experimental result that MTPs are not always present, we find that isotropic faceting conditions will prevent the formation of these particles.

The thermodynamic competition between the different particle types does not imply that MTPs will occur only below a certain size. For an MTP to transform into a single crystal would require extensive atomic rearrangements which would be kinetically forbidden. Once formed, an MTP can in principle grow indefinitely, as evidenced by the experimental observation of particles in the micrometre size range (see, for example, Disgurd et al. 1976). Therefore kinetic or thermodynamic effects in the early stages of growth may determine the final distribution of particle morphologies. However, certain experiments should be affected by the relative thermodynamics. For example, different behaviour would be expected in equilibrium growth from the vapour phase, during Ostwald ripening or in the depression of the melting point.

The presence of any surface impurities may be critical to the thermodynamics of MTPs. The free energy of adsorption is negative and normally quite large. Its prime effect would appear to be to encourage faceting (Blakeley and Somorjai 1977, Flytzani-Stephanopoulos, Wong and Schmidt 1977), possibly magnifying any variations in $\gamma_{\mathrm{s}}$ by reducing the mean level (Rhead and McLean 1964). The effects on MTPs will therefore be mixed. The surface stress of the adsorption is more likely to be of importance as it could easily be large and negative. For instance, a monolayer of silver atoms on the surface of nickel particles would be expected to have a negative $g$. This type of effect may possibly be of significance in bimetallic catalysts.

\section{ACKNOWLEDGMENTS}

We thank Dr. D. J. Smith for the use of the Cambridge High Resolution High Voltage Electron Microscope. This facility, as well as the research project into the structure of MTPs which supports one of us (L.D.M.), is financed by the SERC. We would especially like to thank Dr. E. Yoffe for the inhomogeneous strain solution for icosahedral MTPs given in $\S 2.3$.

\section{REFERENCES}

Atlpress, J. G., and Sanders, J. V., 1967, Surf. Sci., 7, 1.

BAGLEY, B. G., 1965, Nature, Lond., 208, 674.

Blakeley, D. W., and SomorJai, G. A., 1977, Surf. Sci., 65, 419.

Chadwick, G. A., and Kirchner, H. O. K., 1970, Phil. Mag., 22, 447.

Cherns, D., and Stowell, M. J., 1975, Thin Solid Films, 29, 127.

Coulomb, P., 1978, J. Microsc. Spectrosc. Electron., 3, 295.

Disgurd, C., Maurin, M. G., and Roberts, J., 1976, Met Corres Ind 51, 255, 320.

Eshelby, J. D., 1952, Phil. Trans. R. Soc., A, 244, 87 ; 1956, Solid State Physics, Vol. 3, edited by F. Seitz and D. Turnbull (New York, London: Academic Press), p. 79.

Flytzani-Stephanopoulous, M., Wong, S., and Schmidt, L. D., 1977, J. Catal., 49, 51.

Fukano, Y., and Wayman, C. M., 1969, J.appl. Phys., 40, 1656. 
Hayashi, T., Ohno, T., ShIgekr, Y., and Uyeda, R., 1977, Japan J.appl. Phys., 16, 705.

Heinemann, K., Yacaman, M. J., Yang, C. Y., and Poppa, H., 1979, J. Crystal Growth, 47, 177.

Ino, S., 1966, J. phys. Soc. Japan, 21, 346 ; 1969, Ibid., $27,941$.

Ino, S., and OGAWA, T., 1967, J. phys. Soc. Japan, 22, 1369.

Linford, R. G., 1973, Solid State Surface Science II (London: Marcel Dekker), p. 1.

Marks, L. D., 1984, Phil. Mag. A, 49, 81.

Marks, L. D., and HowIe, A., 1979, Nature, Lond., 282, 196.

Marks, L. D., and Smith, D. J. 1981, J., Crystal Growth, 54, 425; 1983, J. Microsc. $130,249$.

Ohyo, T. S., and Yamauchi, K., 1981, Japan J. appl. Phys., 20, 1385.

Rhead, G. E., and MoLean, M., 1964, Acta metall., 12, 401.

ROyal SocIETy, 1969, Symbols, Signs and Abbreviations for British Scientific Publications (London: The Royal Society).

Saito, Y., Yatsuya, S., Mmama, K., and Uyeda, R., 1978, Japan J. appl. Phys., $17,1149$.

Vermaak, J. S., Mays, C. W., and Kuhlmann-Wilsdorf, D., 1968, Surf. Sci., 12, 128.

Volterra, V., 1907, Annls scient. Éc. norm. sup., Paris (Sér. 3), 24, 401.

DE WIT, R., 1969, Fundamental Aspects of Dislocation Theory, Vol. 1, edited by J. A. Simmons, R. de Wit and R. Bullough, NBS Spec. Publ. 317 (Washington : National Bureau of Standards), pp. 651, 677-680; 1972, J. Phys. C, 5, 529.

Yacaman, M. J., Heinemann, K., YANG, C. Y., and Poppa, H., 1979, J. Crystal Growth, 47, 187.

$\mathrm{Y}_{\mathrm{ANG}}$, C. Y., 1979, J. Crystal Growth, 47, 274.

Yang, C. Y., Yacaman, M. J., and HeinemanN, K., 1979, J. Crystal Growth, 47, 283. 